\title{
AVALIAÇÃO DO NÍVEL RESIDUAL TEÓRICO E EXPERIMENTAL DE ENDOSULFAN EM ÓLEO DE SOJA (Glycine max. (L) Merrill) *
}

\author{
CÉLIA MARIA DIAS CORRÊA** \\ JORGE JOSÉ DO VALE OLIVEIRA*** \\ VALDEMAR LUIZ TORNISIELO ****
}

\begin{abstract}
Visou-se quantificar teórica e experimentalmente os níveis residuais de Endosulfan e seus principais metabólitos em óleo de soja e avaliar a confiabilidade dos valores teóricos em relação ao experimental. A soja foi cultivada no campo nos sistemas manejo integrado de pragas (MIP) e convencional. Extraiu-se o óleo de soja utilizando o solvente hexano em extrator Soxhlet. Determinaram-se as concentrações do Endosulfan no grão e no óleo, mediante cromatografia em fase gasosa, com detector de captura de elétrons $\left(\mathrm{Ni}^{63}\right)$. A concentração de Endosulfan no óleo foi obtida teoricamente multiplicando-se a relação entre a massa grão/óleo pela concentração deste inseticida encontrada experimentalmente no grão. A relação percentual da concentração de Endosulfan no óleo, entre os valores teórico/experimental, enquadraram-se na faixa de $94 \%$ a $114 \%$ e $81 \%$ a $92 \%$, respectivamente, para os sistemas MIP e convencional. Como a média geral dos níveis de Endosulfan, calculada teoricamente, alcançou $94 \%$ do valor obtido experimentalmente concluiu-se que o cálculo teórico pode ser utilizado para determinar os níveis deste inseticida no óleo de soja.
\end{abstract}

PALAVRAS-CHAVE: ÓLEO DE SOJA; ENDOSULFAN - NÍVEL RESIDUAL.

* $\quad$ Extraído da dissertação apresentada pela primeira autora ao Centro de Energia Nuclear na Agricultura (CENA), Universidade de São Paulo ( USP), para obtenção do Título de Mestre em Ciências.

** Engenheira Agrônoma, M.Sc., Departamento de Ecotoxicologia, CENA/USP, Piracicaba, SP. (e-mail: cdcorrea@esalq.usp.br).

*** Químico, Pesquisador Científico, Instituto de Tecnologia de Alimentos (ITAL), Campinas, SP. (e-mail: jorgejvo@ital.org.br).

**** Professor Doutor, Departamento de Ecotoxicologia, CENA/USP, Piracicaba, SP. (e-mail: vltornis@cena.usp.br). 


\section{INTRODUÇÃO}

O Endosulfan, inseticida desenvolvido pela Hoechst Aktiengesellschaft na Alemanha, foi introduzido no mercado em 1957 com o nome comercial de Thiodan (GOEBEL et al., 1982; STUMPF \& ABHAVER, 1986). O ingrediente ativo do Endosulfan consiste na mistura de dois isômeros, sendo $70 \%$ de $\alpha$-endosulfan e $30 \%$ de $\beta$-endosulfan. Entre os produtos derivados do metabolismo do Endosulfan em mamíferos, o sulfato de endosulfan é o principal metabólito devido à sua importância toxicológica. A Ingestão Diária Aceitável (IDA) de Endosulfan total (somatório de Endosulfan alfa, beta e sulfato) (Figura 1) para o homem foi estabelecida, em 1989, pelo Joint Meeting on Pesticide Resídue (JMPR) em 0,006 mg/kg de peso corpóreo (DEEMA et al., 1966; DOROUGH \& GIBSON, 1972; LEHR, 1992; STUMPF \& ABHAVER, 1986; FAO/WHO, 1992).

A Legislação Brasileira de Resíduos de Pesticidas vigente estabelece para resíduo de Endosulfan em soja limite máximo de resíduo de $1 \mu \mathrm{g} \cdot \mathrm{g}^{-1}$ e período de carência de 30 dias (BRASIL, 1995).

\section{FIGURA 1 - ESTRUTURAS MOLECULARES DO $\alpha$-ENDOSULFAN (A), $\beta$-ENDOSULFAN (B) E SULFATO DE ENDOSULFAN (C)}

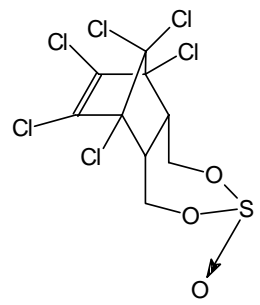

(A)

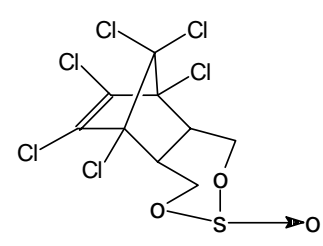

(B)

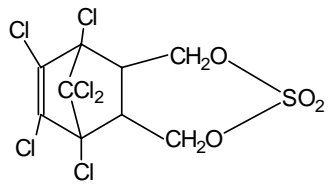

(C)

O Brasil é responsável por cerca de $20 \%$ da produção mundial de soja, sendo o segundo maior produtor de oleaginosas. Destaca-se também como o maior exportador mundial de farelo de soja e o segundo maior exportador de soja e óleo de soja. Praticamente durante todo o seu ciclo a cultura da soja está sujeita ao ataque de insetos. Para combatê-los e viabilizar a produção desta cultura torna-se necessário a aplicação de agrotóxicos. O Endosulfan é muito utilizado para esta finalidade, devido à 
sua eficiência no combate aos insetos (EMBRAPA, 1996; ABIOVE, 1998).

Existem métodos utilizados para determinação de Endosulfan em soja e óleo de soja, cujos resultados apresentam recuperações na faixa de $70 \%$ a $120 \%$, sendo valores aceitos internacionalmente (LUKE et al., 1975; HORWITZ, 1980).

Na quantificação dos isômeros e do metabólito do Endosulfan é empregada a cromatografia em fase gasosa com detector de captura de elétrons $\left(\mathrm{Ni}^{63}\right)$. Para determinação deste inseticida no óleo emprega-se a mesma técnica precedida da etapa de extração do óleo dos grãos da soja. Este procedimento é demorado por envolver duas etapas e, além disso, quando se determina experimentalmente a concentração de Endosulfan no grão, paralelamente, também se quantifica sua concentração no óleo.

Os objetivos deste trabalho foram quantificar teórica e experimentalmente os níveis de Endosulfan em óleo de soja e avaliar a confiabilidade dos valores teóricos em relação aos valores obtidos experimentalmente.

\section{MATERIAL E MÉTODOS}

2.1 SOJA

A variedade da soja utilizada neste experimento foi a IAC 8-2, cultivada em campo experimental do Departamento de Genética, Escola Superior de Agronomia Luiz de Queiroz (ESALQ/USP), em Piracicaba, São Paulo. O plantio da soja foi realizado em março e a colheita efetuada em julho de 1997.

\subsection{DEMARCAÇÃO NO CAMPO}

O experimento foi implantado em solo latossolo roxo (LR) e a área total, isenta de qualquer aplicação anterior de Endosulfan, foi dividida em três parcelas, sendo uma reservada para cultivo da soja controle (isenta da aplicação de Endosulfan) e as outras duas reservadas para o cultivo da soja por meio do manejo integrado de pragas (MIP) e do sistema convencional. Cada parcela apresentou cerca de $300 \mathrm{~m}^{2}$ e população de 40 plantas $/ \mathrm{m}^{2}$, sendo $50 \mathrm{~cm}$ entre linhas e $5 \mathrm{~cm}$ entre plantas. A bordadura deixada foi de $2,5 \mathrm{~m}$, com a finalidade de evitar contaminação entre os tratamentos. 


\subsection{APLICAÇÃO DE ENDOSULFAN (THIODAN 35 CE)}

$\mathrm{Na}$ aplicação de Endosulfan foi utilizado o produto comercial THIODAN 35 CE (1,25 L/ha), recomendado pela EMBRAPA (1996). Para o MIP e sistema convencional foram aplicadas, respectivamente, 1 e 3 doses de 371 g.i.a./ha.

\subsection{AMOSTRAGEM E HOMOGENEIZAÇÃO}

Utilizando-se da pós-colheita manual, os grãos de soja foram separados das vagens secas, homogeneizados em processador e acondicionados em frascos de vidro de $500 \mathrm{~mL}$. Os frascos foram fechados com tampas envolvidas em papel alumínio e armazenados em freezer à temperatura de $-18^{\circ} \mathrm{C}$ até a ocasião das análises.

\subsection{EXTRAÇÃO DO ÓLEO}

A extração do óleo foi realizada com hexano grau pesticida em extrator Soxhlet, sob condições de obtenção comercial de óleo, segundo metodologia da AOCS, método Ac 3.11 (AOCS, 1983). O procedimento de extração do óleo constou das seguintes etapas:

- cerca de $5 \mathrm{~g}$ de grãos de soja homogeneizados foram pesados em papel filtro, dobrado de maneira a não ocorrer perda da amostra e colocado no interior de um cartucho;

- o cartucho foi introduzido no extrator Soxhlet de tal forma que o mesmo ficasse totalmente submerso em hexano durante a extração;

- foram adicionados $150 \mathrm{~mL}$ de hexano, mantendo-se volume constante durante a extração, evitando que houvesse perda significativa do solvente, devido à evaporação;

- concluído o período de extração de 12 horas e após resfriamento, o balão de fundo chato (tarado e pesado previamente em balança semianalítica de duas casas decimais antes da extração), contendo óleo e hexano, foi levado ao rotavapor e mantido a $40^{\circ} \mathrm{C}$ até secura;

- leve fluxo de ar comprimido foi usado para facilitar a remoção de qualquer solvente remanescente;

- $\quad$ para não comprometer a pesagem do óleo, as transferências do balão foram feitas com auxílio de papel toalha impedindo-se a contaminação deste com a gordura da mão. O balão foi levado até a estufa $\left(80^{\circ} \mathrm{C}\right)$ pelo período de 2 horas e depois transferido para dessecador com sílica gel até seu resfriamento à temperatura ambiente e, finalmente, pesado em balança; 
- calculou-se o peso do óleo subtraindo-se o peso do balão mais o óleo menos o peso do balão. A percentagem do óleo na soja foi calculado multiplicando-se a massa do óleo por 100 e o resultado dividido pela massa do grão.

\subsection{PADRÕES, SOLVENTES E REAGENTES}

Soluções de $100 \mathrm{mg} / \mathrm{L}$ de $\alpha$-endosulfan, $\beta$-endosulfan e sulfato de endosulfan (todos com pureza acima de 99,0\%) foram preparadas em hexano grau pesticida (Lichrosolv) e diluídas com o mesmo solvente para concentrações de $10,0 \mathrm{mg} / \mathrm{L}, 1,0 \mathrm{mg} / \mathrm{L}, 0,1 \mathrm{mg} / \mathrm{L}, 0,01 \mathrm{mg} / \mathrm{L}, 0,001 \mathrm{mg} / \mathrm{L}$. Os demais reagentes e solventes utilizados foram: $\mathrm{NaCl}$ (tratado previamente em mufla durante 8 horas a $450 \stackrel{\circ}{\circ}$ ), $\mathrm{Na}_{2} \mathrm{SO}_{4}$ (tratado previamente em mufla durante 8 horas a $650^{\circ} \mathrm{C}$ ), acetona grau pesticida (Lichrosolv) e diclorometano grau pesticida (Lichrosolv).

\subsection{MÉTODO DE ANÁLISE}

Utilizou-se o método de LUKE et al. (1975) para determinação de resíduos de Endosulfan em soja. Em $10 \mathrm{~g}$ de amostra pesada em Erlenmeyer foram adicionados $100 \mathrm{~mL}$ de acetona agitando-se 0 Erlenmeyer durante 1 hora em agitador mecânico. O extrato foi filtrado em funil Büchner com papel de filtro Whatman $n .5$ e recolhido em kitasato acoplado à bomba de vácuo. O filtrado foi transferido para balão volumétrico de $200 \mathrm{~mL}$, com auxílio de funil de vidro e papel de filtro Whatman n. 5, previamente tratado para impedir que qualquer material sólido pudesse permanecer no extrato. Retirou-se alíquota de $20 \mathrm{~mL}$ do extrato, a qual foi transferida para funil de separação de $500 \mathrm{~mL}$, sendo acrescentados $40 \mathrm{~mL}$ de solução aquosa saturada com NaCl e em seguida $100 \mathrm{~mL}$ de mistura de hexano : diclorometano $(50: 50 \mathrm{v} / \mathrm{v})$. Agitou-se o funil vigorosamente durante 5 minutos e abriu-se a torneira de teflon para a saída do vapor colocando-se o funil em suporte até a separação das fases orgânica e aquosa. A fase aquosa, porção inferior, foi transferida para outro funil de separação de $500 \mathrm{~mL}$ e a fase orgânica reservada. Adicionaram-se $40 \mathrm{~mL}$ de diclorometano à fase aquosa agitando-se vigorosamente a mistura por 5 minutos, a qual foi deixada decantar até nova separação de fases. Obteve-se nova fase orgânica na porção inferior do balão, a qual foi adicionada à fase orgânica reservada descartando-se a fase aquosa. A mistura das fases orgânicas foi filtrada em funil com sulfato de sódio, previamente seco em mufla, para a retirada de eventual resíduo de água do processo de separação.

$\mathrm{O}$ extrato foi recolhido em balão de fundo chato de $125 \mathrm{~mL}$ e 
levado até o rotavapor em temperatura de $40^{\circ} \mathrm{C}$, sendo evaporado até quase secura. Ao extrato foi adicionado hexano GP com pipeta Pasteur tomando-se cuidado na lavagem das paredes do balão. $O$ extrato foi transferido com pipeta Pasteur para tubo concentrador e corrigido para volume final de $5 \mathrm{~mL}$ (efetuando-se quando necessário, nova diluição para obter concentração na faixa de linearidade do detector) para ser usado na determinação e identificação dos resíduos. Todas as transferências foram feitas quantitativamente lavando-se cuidadosamente cada recipiente com pequenas porções do solvente utilizado, a fim de minimizar perdas de resíduo junto à vidraria.

Para quantificação foi utilizado cromatógrafo a gás Varian 3.700 (CG), com coluna de vidro de 1,5 m de comprimento, diâmetros interno de $2 \mathrm{~mm}$ e externo de $6 \mathrm{~mm}$, empacotada com a fase estacionária $5 \%$ SE-30 (100-120) Supelcoport e equipado com detector de captura de elétrons ( $\mathrm{DCE})\left(\mathrm{Ni}^{63}\right)$. As condições otimizadas, previamente, envolveram: injetor a $240{ }^{\circ} \mathrm{C}$, coluna com temperatura inicial de $150{ }^{\circ} \mathrm{C}$, durante 5 minutos, rampa de temperatura de $1^{\circ} \mathrm{C} / \mathrm{min}$ até $230^{\circ} \mathrm{C}(5$ minutos), detector a $320^{\circ} \mathrm{C}$ e atenuação de 8.10 , registrador com $2 \mathrm{mV}$ e velocidade de carta de $5 \mathrm{~cm} / \mathrm{h}$, fluxo médio de nitrogênio de $35 \mathrm{psi}$ (psi = $10 \mathrm{lbs} /$ polegada quadrada ou 6,8046 atm).

A metodologia utilizada para determinação de Endosulfan no óleo foi a mesma utilizada no grão, com a seguinte diferença: pesou-se $1 \mathrm{~g}$ do óleo, efetuou-se a extração e o volume final foi retomado em $5 \mathrm{~mL}$ de hexano.

\subsection{VALIDAÇÃO DE METODOLOGIA E CONFIRMAÇÃO}

Para validar a metodologia amostras de soja, isentas de Endosulfan, foram fortificadas ao nível de $0,10 \mathrm{mg} / \mathrm{kg}$ e $0,01 \mathrm{mg} / \mathrm{kg}$ para $\alpha$ e $\beta$ endosulfan. Com o sulfato de endosulfan as fortificações ocorreram em níveis de $0,50 \mathrm{mg} / \mathrm{kg}$. Foram construídas curvas padrão na faixa de linearidade de $0,005 \mathrm{ng}$ a $0,020 \mathrm{ng}$ para $\alpha$-endosulfan e $\beta$-endosulfan, com coeficiente de linearidade de 0,9975 e 0,9951, respectivamente. Para sulfato de endosulfan a faixa de linearidade foi de $0,05 \mathrm{ng}$ a $0,20 \mathrm{ng}$, com coeficiente de linearidade de 0,9947.

Para confirmação dos picos de Endosulfan foi utilizado cromatógrafo a gás Varian 3.400, equipado com $\mathrm{DCE}\left(\mathrm{Ni}^{63}\right)$ e coluna megabore DB-5 (30 m x 0,53 mm x 0,85 $\mu \mathrm{m})$. As condições cromatográficas foram: temperaturas de detector $300^{\circ} \mathrm{C}$ e injetor $250^{\circ} \mathrm{C}$, programação de temperatura de coluna de $180^{\circ} \mathrm{C}$, durante 1 minuto, elevando $6^{\circ} \mathrm{C} / \mathrm{min}$ até $250^{\circ} \mathrm{C}$ e permanecendo nesta temperatura por 5 minutos. Fluxo de nitrogênio de 18 psi; velocidade da carta de $20 \mathrm{~cm} / \mathrm{h}$, atenuação 8.10 e registrador operando a $1 \mathrm{mV}$. 


\subsection{CÁLCULO TEÓRICO DE ENDOSULFAN NO ÓLEO}

Para calcular teoricamente a concentração de Endosulfan no óleo empregou-se a seguinte equação:

Sendo:

$$
\text { Cto }=\text { Ceg. } \mathrm{Mg} / \mathrm{Mo} \text {. }
$$

Cto = Concentração teórica de Endosulfan no óleo $(\mathrm{mg} / \mathrm{kg})$; Ceg = Concentração experimental de Endosulfan no grão $(\mathrm{mg} / \mathrm{kg})$; $\mathrm{Mg}=$ Massa do grão.

Mo = Massa do óleo extraído por Soxhlet.

\section{RESULTADOS E DISCUSSÃO}

Os cromatogramas dos extratos dos grãos, isentos de Endosulfan como os do óleo extraído desta amostra, não apresentaram picos interferentes nos tempos de retenção dos isômeros do endosulfan, $\alpha$ e $\beta$, nem do metabólito sulfato de endosulfan.

As recuperações do $\alpha$-endosulfan, $\beta$-endosulfan e sulfato de endosulfan nos grãos enquadraram-se na faixa de $84 \%$ a $120 \%$ nos níveis de fortificação de $0,5 \mathrm{mg} / \mathrm{kg}, 0,1 \mathrm{mg} / \mathrm{kg}$ e $0,01 \mathrm{mg} / \mathrm{kg}$. Não houve necessidade de fortificar o óleo com o Endosulfan e verificar a recuperação, porque a quantidade de Endosulfan encontrada no grão solubilizou-se no óleo, após extração deste.

Os resultados das análises de $\alpha$-endosulfan, $\beta$-endosulfan e sulfato de endosulfan, referentes à soja tratada pelo sistema MIP e convencional e os respectivos valores teórico e experimental encontramse na Tabela 1.

Verificou-se (Tabela 1) que a relação de massa entre o grão e o óleo extraído deste manteve-se praticamente constante, independente da aplicação do inseticida na cultura da soja.

Conforme os resultados obtidos no presente trabalho, a massa do óleo correspondeu em média a $16 \%$ da massa do grão. Isto representa concentração de Endosulfan no óleo cerca de seis vezes maior em relação ao grão, considerando que o inseticida tenha sido todo extraído e se solubilizado no óleo. De acordo com os resultados experimentais de extração de Endosulfan nas amostras ocorreu mesma proporcionalidade (1/6) entre as concentrações do inseticida no grão/óleo. Os níveis de Endosulfan encontrados no óleo e no grão da soja tratada pelo sistema convencional foram maiores do que os do sistema MIP, entretanto a relação 
de proporcionalidade entre as concentrações do inseticida no grão/óleo foram as mesmas. Estes resultados são coerentes com a propriedade lipofílica do Endosulfan. Alguns trabalhos também confirmam a presença de Endosulfan em óleos comestíveis extraídos de várias matérias-primas, como arroz, milho, girassol, algodão e sobretudo soja (MAINI et al., 1982; YOKOMIZO et al., 1984; RAJ et al., 1990).

\section{TABELA1 - NÍVEIS DE ENDOSULFAN (mg/kg) TEÓRICO E EXPERIMENTAL (EXTRAÍDO POR SOXHLET + LUKE) EM AMOSTRAS DE ÓLEO}

\begin{tabular}{|c|c|c|c|c|c|}
\hline Tratamento & $\begin{array}{c}\text { Massa } \\
\left(\mathrm{Gr}^{\mathrm{a}} \mathrm{os} / \mathrm{leo}\right) \\
(\mathrm{g})\end{array}$ & $\begin{array}{l}\text { GR^OS } \\
(\mathrm{LUKE})^{*} \\
(\mathrm{E} \alpha+\mathrm{E} \beta)\end{array}$ & $\begin{array}{c}\text { leo } \\
(\mathrm{Te} \text { rico) } \\
(\mathrm{E} \alpha+\mathrm{E} \beta)\end{array}$ & $\begin{array}{c}\text { leo } \\
{\text { (Soxhlet+LUKE })^{* *}}_{(E \alpha+E \beta)}\end{array}$ & $\begin{array}{c}\text { Rela }{ }^{\mathrm{a}} 0 \\
\text { Te rico/Experimental } \\
(\%)\end{array}$ \\
\hline \multirow{3}{*}{ Testemunha } & $4.99 / 0.84$ & $\mathrm{Nd}$ & - & - & - \\
\hline & $4.99 / 0.76$ & $\mathrm{Nd}$ & - & - & - \\
\hline & $5.02 / 0.80$ & $\mathrm{Nd}$ & - & - & - \\
\hline MØdia 1 & $5.00 / 0.80$ & - & - & - & - \\
\hline \multirow[t]{6}{*}{ MIP } & $5.04 / 0.83$ & 0.027 & 0.16 & 0.14 & 114 \\
\hline & $5.00 / 0.82$ & 0.027 & 0.16 & 0.14 & 114 \\
\hline & $5.05 / 0.81$ & 0.026 & 0.16 & 0.17 & 94 \\
\hline & $5.01 / 0.82$ & 0.023 & 0.14 & 0.13 & 107 \\
\hline & $4.99 / 0.81$ & 0.019 & 0.12 & 0.11 & 109 \\
\hline & $5.01 / 0.83$ & 0.019 & 0.11 & 0.11 & 100 \\
\hline MØdia 2 & $5.02 / 0.82$ & 0.023 & 0.14 & 0.13 & 107 \\
\hline \multirow[t]{5}{*}{ Convencional } & $5.01 / 0.87$ & 0.034 & 0.20 & 0.23 & 87 \\
\hline & $4.98 / 0.80$ & 0.034 & 0.21 & 0.23 & 92 \\
\hline & $5.05 / 0.82$ & 0.034 & 0.21 & 0.26 & 81 \\
\hline & $5.11 / 0.81$ & 0.034 & 0.21 & 0.26 & 81 \\
\hline & $5.03 / 0.82$ & 0.037 & 0.23 & 0.27 & 84 \\
\hline Mødia 3 & $5.04 / 0.82$ & 0.037 & 0.23 & 0.27 & 81 \\
\hline MØdia (2 + 3) & $5.03 / 0.82$ & 0,035 & 0,21 & 0,25 & 94 \\
\hline
\end{tabular}

$(E \alpha+E \beta)=$ Endosulfan $(\alpha+\beta)$.

* Extração de Endosulfan experimentalmente pelo método de LUKE et al., 1975.

** Extração do óleo da soja em Soxhlet, seguido da quantificação experimental de Endosulfan no óleo pelo método do LUKE et al., 1975.

$\mathrm{Nd}=$ não-detectado, abaixo do limite de quantificação do método $\alpha$-endosulfan $(0.01 \mathrm{mg} / \mathrm{kg})$ e $\beta$-endosulfan $(0.01 \mathrm{mg} / \mathrm{kg})$. 
A concentração média, calculada teoricamente, do Endosulfan no óleo de soja em relação ao obtido experimentalmente no mesmo óleo foi de $94 \%$.

Não foram detectados resíduos de sulfato de endosulfan nas amostras de grão e óleo, dentro do limite de quantificação do método $(0,5 \mathrm{mg} / \mathrm{kg})$. Conforme pode ser verificado pela Tabela 1 , apenas os isômeros $\alpha$-endosulfan e $\beta$-endosulfan foram detectados em ambas as amostras. A não-detecção do principal metabólito do Endosulfan está associada ao fato dos isômeros não terem sofrido reação de oxidação, processo químico diretamente ligado à exposição da molécula ao oxigênio.

De acordo com LAWSON (1995) os óleos naturais e gorduras de origem vegetal contêm pequenas quantidades de substâncias capazes de inibir oxidação. Dentre os antioxidantes universalmente distribuídos, o alfa-tocoferol é o mais importante. Segundo BOBBIO \& BOBBIO (1995) os tocoferóis são fortes redutores e, portanto, considerados como antioxidantes naturais.

Os tocoferóis ocorrem na natureza na forma de óleo viscoso, de coloração amarela, insolúvel em água e solúvel em álcool, éter e gorduras. São estáveis ao calor, ácidos e álcalis, mas sensíveis ao oxigênio e a outros oxidantes, principalmente quando em presença de metais pesados. Também são muito estáveis no processamento de alimentos, mesmo em temperaturas elevadas (até $200^{\circ} \mathrm{C}$ em ausência de oxigênio) (RIBEIRO, 1950).

Com relação à ação dos óleos na inibição da oxidação, antigos estudos efetuados com óleo de gergelim verificaram sua capacidade de atuar no processo de inibição da epoxidação de ciclodienos. Vale lembrar que o Endosulfan é um ciclodieno que não se degrada por processos de epoxidação (O'BRIEN, 1967).

Verifica-se pela Figura 1 que os níveis de $\alpha$-endosulfan foram menores do que $\beta$-endosulfan, embora LEHR (1992) defina o ingrediente ativo do Endosulfan como mistura de dois isômeros, sendo $70 \%$ $\alpha$-endosulfan e $30 \%$ de $\beta$-endosulfan. Este resultado pode ser explicado pela susceptibilidade do $\alpha$-endosulfan em sofrer reação de descloração fotoquímica, enquanto que o $\beta$-endosulfan se apresenta como molécula mais estável (LEHR, 1993).

\section{CONCLUSÃO}

Conhecendo-se experimentalmente a concentração de Endosulfan no grão e a relação entre a massa do grão e do óleo pode-se calcular teoricamente a concentração deste inseticida no óleo. Independente do 
tratamento (MIP ou convencional) a que a soja foi submetida e do nível de Endosulfan encontrado no grão, a diferença entre a concentração do Endosulfan no óleo, determinada experimentalmente e calculada teoricamente, não foi significativa.

\section{FIGURA 1 - RESULTADO MÉDIO DE ENDOSULFAN NO ÓLEO NOS SISTEMAS MIP E CONVENCIONAL}

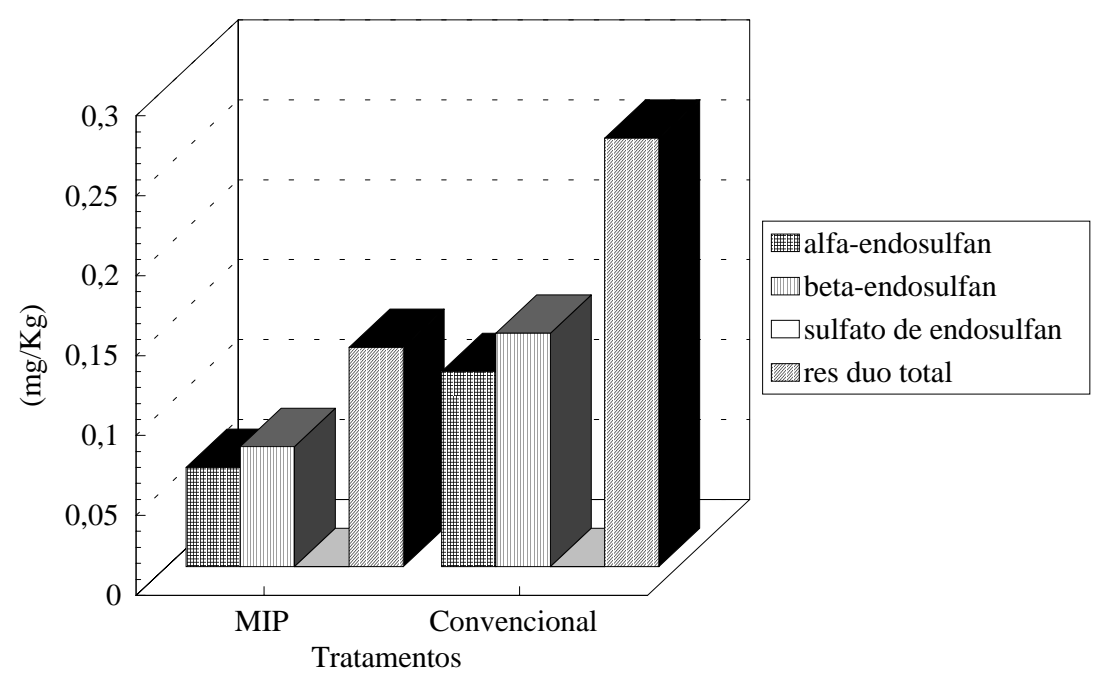

\section{Abstract \\ EVALUATION OF THE THEORETICAL AND EXPERIMENTAL RESIDUAL LEVELS OF ENDOSULFAN IN SOYBEAN (Glycine max. (L) Merrill) OIL}

The objective of this study was to quantify theoretically and experimentally the residual levels of Endosulfan and main metabolites in soybean oil and to evaluate the reliability of the theoretical values in relation to the experimental values. The soybean was grown in the fields in the pest integrated management (PIM) and conventional systems. The soybean oil was extracted by using hexane as solvent in Soxhlet extractor. The Endosulfan concentrations were determined in the grains and oil by means of gas chromatography equipped with electron capture detector $\left(\mathrm{Ni}^{63}\right)$. The Endosulfan concentration in soybean oil was theoretically obtained by multiplying the relationship between the grain mass/ soybean oil by this insecticide concentration determined experimentally in the grain. The percents relationship of the concentration of Endosulfan in the soybean oil between the theoretical/experimental values were in the range of $94 \%$ to $119 \%$ and $81 \%$ to $92 \%$, 
respectively, for the PIM and conventional system. As the general average levels of Endosulfan, theoretically calculated, reached $94 \%$ of the value obtained experimentally it was concluded that the theoretical calculation could be used for determination this insecticide levels in the soybean oil.

KEY-WORDS: SOYBEAN OIL; ENDOSULFAN - RESIDUAL LEVELS.

\section{REFERÊNCIAS}

1 AOCS. American Oil Chemists Society. Official and tentative methods. $3^{\text {rd }}$ ed. Champaing, 1983. (Method Ac 3.11).

2 ABIOVE. Associação Brasileira das Industrias de Óleos Vegetais. Disponível em: <http://www.abiove.com.br>. Acesso em 1998.

3 BOBBIO, P.A.; BOBBIO, F.O. Química do processamento de alimentos. São Paulo: Varela, 1995. p. 46-48.

4 BRASIL. Ministério da Saúde. Relação de substâncias para uso domissanitário: portarias do Ministério da Saúde. São Paulo: International Life Sciences Institute (ILSI), 1995. 716 p.

5 DEEMA, P.; THOMPSON, E.; WARE, G.W. Metabolism, storage and excretion of ${ }^{14} \mathrm{C}$-Endosulfan in mouse. Journal of the Economic Entomology, v. 59, p. 546-550, 1966.

6 DOROUGH, H.W.; GIBSON, J.R. Chlorinated insecticide residues in cigarettes purchased 1970-72. Environmental Entomology, v.1, n.6, p. 739-743, 1972.

7 EMBRAPA. Empresa Brasileira de Pesquisa Agropecuária. Recomendações técnicas para a cultura da soja na região Central do Brasil 1996/97. Londrina: EMBRAPA-CNPSo, 1996. $164 \mathrm{p}$.

8 FAO/WHO. Food and Agriculture Organization. World Health Organization. International program on chemical safety. In: JOINT MEETING ON PESTICIDE RESIDUES (JMPR), Genebra, 1991. Genebra: WHO, 1992. 35 p. (WHO/PCS/92.9).

9 GOEBEL, H.; GORBACH, S.; KNAUF, W.; RIMPAU, R.H.; $\mathrm{HÜTTENBACH}, \mathrm{H}$. Properties, effects, residues, and analytics of the insecticide Endosulfan. New York: Springer-Verlag, 1982. 174 p. (Residues Reviews, 83). 
10 HORWITZ, W. (Ed.). Official Methods of Analysis of the Association of Official Analytical Chemists. $13^{\text {th }}$ ed. Washington, D.C.: AOAC, 1980. p. 466-496.

11 LAWSON, H. Food oils and fats: technology, utilization and nutrition. New York: Chapman \& Hall, 1995. p. 15-27.

12 LEHR, W. Statement concerning soil metabolism and mobility of Endosulfan (Hoe 002671). Frankfurt: HOECHST, 1992. p.1-8. (Report, PSR-WLO2/92).

13 LEHR, W. Statement concerning the chemical classification of Endosulfan. Frankfurt: HOECHST, 1993. p.1-3. (Report, PSR93/034).

14 LUKE, M.A.; FROBERG, J.E.; MASUMOTO, H.T. Extraction and clean-up of organochlorine, organophosphate, organonitrogen and hidrocarbon pesticides in produce for determination by gas-liquid chromatography. Journal of the Association of Official Analytical Chemists, v.58, n.5, p.1020-1026, 1975.

15 MAINI, O.S.; DEWAN, R.S.; AGNIHOTRI, N.P.; JAIN, H.K.; SRIVASTAVA, K.P. Residues from Endosulfan application on cotton crop. Journal of Entomological Research, v.6, n.1, p.90-95, 1982.

16 O'BRIEN, R.D. Insecticides action and metabolism. New York: Academic Press, 1967. p.133-147.

17 RAJ, M.F.; SHAH, P.G.; PATEL, B.K.; TALATI, J.G.; PATEL A.S. Residues of synthetic pyrethroids in cotton seed, oil and lint. Indian Journal of Plant Protection, v.18, n.2, p.191-195, 1990.

18 RIBEIRO, F. Vitaminas: noções fundamentais, teor nos alimentos. 2.ed. São Paulo: Melhoramentos, 1950. p. 114-118.

19 STUMPF, K.; ABHAVER, J. An up-to-date review of the environmental chemistry of Endosulfan. Frankfurt: HOECHST, 1986. 23 p. (Report B 81/86).

20 YOKOMIZO, Y.; MANTOVANI, D.M.B.; ANGELUCCI, E.; PASQUINELLI, S.R.; DESTRO, M.T. Contamination of edible oils and vegetables fats with metals and pesticide residues. Boletim do Instituto de Tecnologia de Alimentos, v.21, n.2, p.203-238, 1984. 